Carolina Araújo dos Santos ${ }^{1}$

Andréia Queiroz Ribeiro ${ }^{1}$

Carla de Oliveira Barbosa Rosa ${ }^{1}$

Rita de Cassia Lanes Ribeiro ${ }^{1}$

${ }^{1}$ Departamento de Nutrição e Saúde, Universidade Federal de Viçosa. Av. Ph Rolfs s/n, Campus Universitário. 36570000 Viçosa MG Brasil. carolaraujors@hotmail.com

\title{
Depressão, déficit cognitivo e fatores associados à desnutrição em idosos com câncer
}

\author{
Depression, cognitive deficit and factors associated with \\ malnutrition in elderly people with cancer
}

Abstract The study sought to analyze the presence of depression, cognitive function, nutritional status and factors associated with malnutrition in the elderly undergoing treatment for cancer. A cross-sectional study was conducted in a cancer clinic in Minas Gerais, which included the application of a sociodemographic and health questionnaire, assessment of depressive symptoms, cognitive function and nutritional status. $96 \mathrm{el}$ derly people were evaluated, 50\% of which were female and the mean age was 70.6 years. Cognitive impairment was identified in $39.6 \%$ and the presence of depression in $17.7 \%$, with a higher number of depressive symptoms in females ( $p=$ 0,017). Moderate malnutrition or suspected malnutrition was diagnosed in $29.2 \%$, severe malnutrition in $14.6 \%$ and $47.9 \%$ had a critical need for nutritional intervention. In multivariate analysis, functional impairment was independently associated with malnutrition (PR: 3.40; CI: 1.23-9.45), the presence of two or more symptoms of nutritional impact (PR: 3.22; CI: 10.03-10.10) and current treatment by chemotherapy (PR: 2.96; CI: 1.16-7.56). The elderly with cancer showed a high prevalence of malnutrition and a need for nutritional intervention. The assessment of depression and cognitive impairment should also be an integral part in addressing this group.

Key words The elderly, Neoplasms, Malnutrition, Depression, Cognition
Resumo Objetiva-se analisar a presença de depressão, a função cognitiva, o estado nutricional e os fatores associados à desnutrição em idosos em tratamento para o câncer. Foi realizado um estudo transversal em um centro oncológico em Minas Gerais que incluiu a aplicação de um questionário sociodemográfico e de saúde, a avaliação de sintomas depressivos, da função cognitiva e do estado nutricional. Foram avaliados 96 idosos, 50\% do sexo feminino e com idade média de 70,6 anos. $O$ déficit cognitivo foi identificado em 39,6\% e a presença de depressão em $17,7 \%$ dos avaliados, com um maior número de sintomas depressivos sexo feminino $(p=0,017)$. Foi diagnosticada desnutrição moderada ou suspeita de desnutrição em $29,2 \%$ dos avaliados, desnutrição grave em 14,6\% e 47,9\% apresentou necessidade de intervenção nutricional crítica. Na análise multivariada foram condições independentemente associadas à desnutrição o déficit funcional (RP: 3,40; IC: 1,23-9,45), a presença de dois ou mais sintomas de impacto nutricional (RP: 3,22; IC: 1,03-10,10) e o tratamento atual por quimioterapia (RP: 2,96; IC: 1,16-7,56). Idosos com câncer apresentaram elevada prevalência de desnutrição e de necessidade de intervenção nutricional. A avaliação da depressão e do déficit cognitivo também devem ser partes integrantes na abordagem deste grupo.

Palavras-chave Idoso, Neoplasias, Desnutrição, Depressão, Cognição 


\section{Introdução}

O envelhecimento é um novo desafio para a saúde pública contemporânea, bem como um fator de risco independente para o câncer ${ }^{1}$. Considerada a segunda causa de morte no Brasil, projeções indicam mais de 500 mil novos casos da doença no país em 2013, e, mundialmente, cerca de 75 milhões indivíduos com tumores em $2030^{2}$. Pessoas idosas representam a maioria dos casos novos e dos óbitos pela doença, indicando a necessidade de uma atenção específica a este grupo e suas particularidades ${ }^{3}$.

A desnutrição é uma condição frequente em indivíduos com câncer, considerada um processo multifatorial associado à evolução tumoral e aos impactos e efeitos colaterais dos tratamentos. Por se relacionar à maior morbimortalidade e complicações, o diagnóstico precoce e a intervenção nutricional adequada são essenciais para melhorar o prognóstico ${ }^{4,5}$. Em relação ao diagnóstico nutricional, o Consenso Brasileiro de Nutrição Oncológica ${ }^{2}$ propôs recentemente a utilização da Avaliação Subjetiva Global Produzida pelo Paciente (ASG-PPP). Trata-se de um método subjetivo, dependente da participação do avaliado, que foi traduzido e validado para a população brasileira ${ }^{6}$. No que diz respeito à avaliação de idosos com câncer, pouco ainda se sabe sobre sua aplicabilidade e quais parâmetros se associariam à maior ocorrência de desnutrição diagnosticada por este novo método.

Além da maior incidência de tumores relacionados ao envelhecimento, em idosos é comum a presença de comorbidades ou problemas de saúde que também podem ter impacto negativo no estado nutricional e na qualidade de vida ${ }^{7}$. Neste aspecto, destaca-se a presença de déficit cogniti$v^{8}{ }^{8}$ e de depressão9.

Alterações cognitivas são comuns em indivíduos com câncer, por razões relacionadas à doença e ao tratamento ${ }^{10}$. Fatores como sofrimento emocional (ansiedade/depressão) e sintomas físicos também podem intensificar o quadro ${ }^{11}$, comprometendo o desempenho de atividades diárias, a interação social, a adesão ao tratamento e a qualidade de vida, já comprometida pelo câncer e pelos impactos da terapia antineoplásica ${ }^{12}$. O déficit cognitivo é descrito ainda como um fator que aumenta o risco de desnutrição, uma vez que pode relacionar-se à diminuição do apetite, redução da habilidade de manipulação e de reconhecimento dos alimentos e dificuldades de mastigação e deglutição ${ }^{13}$. Embora seja um transtorno relatado na literatura, estudos sobre a pre- valência das alterações cognitivas em idosos com câncer ainda são incipientes.

Em relação à depressão, acredita-se que seja o transtorno mental mais comum em idosos, tendo impacto negativo em todos os aspectos da vida ${ }^{14}$. Acredita-se que também seja uma condição frequente em pacientes oncológicos, principalmente por questões relacionadas à gravidade da doença e aos efeitos colaterais dos tratamentos ${ }^{15}$. Quando não tratada, a depressão relaciona-se à maior morbidade e mortalidade, aumento dos custos de assistência ${ }^{16}$, com impacto negativo na qualidade de vida e na motivação quanto à adesão ao tratamento ${ }^{17}$. Associa-se também ao aumento na utilização dos serviços de saúde, à negligência no autocuidado, à adesão reduzida aos regimes terapêuticos e ao maior risco de suicídio ${ }^{18,19}$. Estudos indicam ainda a associação entre a depressão e o menor consumo alimentar, perda de peso e desnutrição $\mathrm{O}^{20,21}$

Sugere-se, ainda, a influência de condições sociodemográficas, de saúde e clínicas no prognóstico e no estado nutricional de idosos ${ }^{22}$. Tais influências, entretanto, são insuficientemente exploradas, uma vez que a literatura ainda é limitada no que diz respeito à aplicabilidade da ASG-PPP para o diagnóstico de desnutrição, especialmente no Brasil.

Assim, o presente estudo teve o objetivo de avaliar a presença de depressão, a função cognitiva, o estado nutricional e os fatores associados à desnutrição em idosos em tratamento oncológico. Especialmente com uma amostra composta exclusivamente por idosos, não encontramos referências nacionais ou internacionais da utilização da ASG-PPP com a investigação destas associações. O estudo dessas relações é relevante no intuito de identificar parâmetros importantes no cuidado ao idoso com câncer e de elucidar possíveis associações que direcionem estratégias de intervenção.

\section{Metodologia}

Trata-se de um estudo transversal com amostra constituída por idosos que realizaram tratamento em um centro oncológico no município de Ponte Nova, Minas Gerais, no período de julho a novembro de 2012. Foram adotados os critérios de inclusão: idade igual ou superior a 60 anos, diagnóstico de câncer confirmado por biópsia e tratamento já iniciado, atendimento em nível ambulatorial e anuência para a participação mediante assinatura do Termo de consentimento Livre e Esclarecido. 
Dos indivíduos cadastrados no serviço $(\mathrm{n}=$ 206), 51,5\% $(\mathrm{n}=106)$ eram idosos. Destes, 98 atenderam aos critérios de inclusão e, considerando duas recusas, a amostra final foi composta por 96 idosos.

O protocolo do estudo incluiu uma entrevista para a obtenção de dados sociodemográficos e de saúde, avaliação do estado nutricional, da função cognitiva e de sintomas depressivos. A entrevista foi realizada por meio da aplicação de um questionário semiestruturado no qual foram coletadas as seguintes informações: dados pessoais; histórico clínico; estado civil; escolaridade; renda per capita e condições de saúde (doenças autorreferidas, percepção da saúde e uso de medicamentos). A coleta de informações complementares, como data do diagnóstico, início, tipo e características do tratamento, foram obtidas diretamente dos prontuários e por consulta ao médico responsável pelo paciente. Após a entrevista, os idosos foram avaliados em relação ao estado nutricional, à função cognitiva e à presença de sintomas depressivos.

O estado nutricional foi avaliado pela Avaliação Subjetiva Global Produzida pelo Paciente (ASG-PPP), versão traduzida e validada para a população brasileira ${ }^{6}$. A primeira parte foi aplicada na forma de entrevista com o idoso e a segunda parte foi preenchida pela nutricionista pesquisadora. A classificação do estado nutricional incluiu as categorias: eutrofia (Estágio A), desnutrição moderada ou suspeita de desnutrição (Estágio B) e desnutrição grave (Estágio C). Foi calculada também a pontuação referente à triagem nutricional, que, quando maior ou igual a nove pontos indica a necessidade de intervenção nutricional crítica.

Para a avaliação da função cognitiva foi aplicado o Mini Exame do Estado Mental (MEEM) ${ }^{23}$, traduzido e validado para a população brasilei$\mathrm{ra}^{24}$. Foram utilizados os pontos de corte propostos por Almeida e Almeida ${ }^{25}: 20$ pontos para indivíduos sem escolaridade e 24 pontos para indivíduos com escolaridade. Idosos com pontuação abaixo do ponto de corte foram diagnosticados com déficit cognitivo.

A avaliação da depressão foi realizada pela Escala de Depressão Geriátrica - Geriatric Depression Scale, versão com 15 itens (GDS-15). Os idosos responderam na forma de entrevista individual a 15 perguntas afirmativas/negativas e a classificação foi realizada segundo a pontuação: 0 a 5 pontos: exame normal; 6 a 10 pontos: quadro depressivo leve; pontuação igual ou superior a 11: provável depressão grave ${ }^{26}$.
Foi realizada análise descritiva para a caracterização da amostra, a partir das medidas de frequência (absoluta e relativa) e medidas de tendência central e dispersão. A comparação entre as pontuações da ASG-PPP, MEEM e GDS-15 segundo o sexo foi realizada pelo teste de Mann-Whitney, uma vez que as variáveis não apresentaram distribuição normal pelo teste de Kolmogorov-Sminorv. Os idosos classificados em desnutrição moderada/suspeita de desnutrição (Estágio B) ou desnutrição grave (Estágio C) foram agrupados em uma única categoria (desnutrição), que foi considerada como variável dependente na análise de associação. A associação entre as variáveis exploratórias e a presença de desnutrição foi verificada na análise bivariada pelo teste do qui-quadrado de Pearson ou pelo teste exato de Fisher, com nível de 5\% de significância. Foram calculadas as razões de prevalência (RP) e os intervalos de confiança de $95 \%$ (IC 95\%) por meio do modelo univariado de regressão de Poisson com ajuste de variância robusto. Para o processo de modelagem final, adotou-se o modelo composto pelas variáveis selecionadas a partir da análise univariada $(\mathrm{p}<0,2)$, sendo consideradas independentemente associadas à desnutrição aquelas que apresentaram associação com a variável resposta com $\mathrm{p}<0,05$. As análises foram realizadas no software STATA (Versão 9.1).

O protocolo do estudo foi aprovado pelo Comitê de Ética em Pesquisa com Seres Humanos da UFV e os idosos que concordaram em participar assinaram o termo de Consentimento Livre e Esclarecido, na presença do familiar/acompanhante.

\section{Resultados}

Participaram do estudo 96 idosos, 50\% do sexo masculino e com idade média de 70,6 anos (DP $=7,8$ anos). A amostra foi composta predominantemente por idosos casados (56,3\%), com até quatro anos de estudo $(78,1 \%)$ e renda per capita inferior a um salário mínimo (57,3\%).

Os tumores de maior prevalência foram os de próstata $(27 \%)$, mama (22\%), pulmão $(9,4 \%)$, esôfago $(9,4 \%)$ e estômago $(8,3 \%)$. Dos idosos avaliados, 49\% apresentavam metástase, 51\% haviam realizado cirurgia oncológica e 35,4\% fizeram radioterapia prévia. Os idosos estavam em sua maioria em tratamento por quimioterapia $(66,7 \%)$ e apresentavam a doença em estágio III ou IV $(83,3 \%)$.

A avaliação nutricional identificou desnutrição moderada ou grave em $43,8 \%$, a presença de 
pelo menos um sintoma de impacto nutricional em $65,7 \%$ e necessidade de intervenção nutricional crítica em 47,9\% dos idosos (Tabela 1).

O déficit cognitivo foi identificado em 39,6\% dos idosos e a presença de depressão foi obser-

Tabela 1. Parâmetros avaliados pela Avaliação Subjetiva Global Produzida pelo Paciente em idosos em tratamento oncológico. Ponte Nova, 2012.

\begin{tabular}{|c|c|c|}
\hline Parâmetros & $\mathbf{n}$ & $\%$ \\
\hline \multicolumn{3}{|l|}{ Sintomas } \\
\hline Sem problemas para se alimentar & 33 & 34, \\
\hline Sem apetite & 40 & 41,7 \\
\hline Náusea & 20 & 20,8 \\
\hline Obstipação & 41 & 42,7 \\
\hline Feridas na boca & 15 & 15,6 \\
\hline $\begin{array}{l}\text { Alimentos com gosto estranho ou sem } \\
\text { gosto }\end{array}$ & 25 & 26, \\
\hline Cheiros enjoam & 23 & 23, \\
\hline Rapidamente se sente satisfeito & 33 & 34,4 \\
\hline Vômito & 17 & 17,7 \\
\hline Diarreia & 11 & 11,5 \\
\hline Boca seca & 47 & 48, \\
\hline Problemas para deglutir & 9 & 9 , \\
\hline Dor & 4 & 4,4 \\
\hline Outros & 2 & 2, \\
\hline \multicolumn{3}{|l|}{ Capacidade funcional } \\
\hline \multicolumn{3}{|l|}{ Atividade no último mês } \\
\hline Normal, sem limitação & 39 & 40 , \\
\hline $\begin{array}{l}\text { Não totalmente normal, mas capaz } \\
\text { de manter quase todas as atividades } \\
\text { normais }\end{array}$ & 24 & 25,0 \\
\hline $\begin{array}{l}\text { Não se sentindo bem para a maioria } \\
\text { das coisas, mas ficando na cama ou } \\
\text { na cadeira menos da metade do dia }\end{array}$ & 15 & 15 , \\
\hline $\begin{array}{l}\text { Capaz de fazer pouca atividade, e } \\
\text { passando a maior parte do tempo na } \\
\text { cadeira ou na cama }\end{array}$ & 13 & 13 , \\
\hline $\begin{array}{l}\text { Bastante tempo acamado, raramente } \\
\text { fora da cama }\end{array}$ & 5 & 5 , \\
\hline \multicolumn{3}{|l|}{ Triagem nutricional } \\
\hline $0-1$ pontos $^{\mathrm{a}}$ & 3 & 3 , \\
\hline $2-3$ pontos $^{\mathrm{b}}$ & 8 & 8 , \\
\hline 4-8 pontos $^{c}$ & 39 & 40, \\
\hline$\geq 9$ pontos $^{\mathrm{d}}$ & 46 & 47 , \\
\hline \multicolumn{3}{|l|}{ Avaliação nutricional subjetiva } \\
\hline Bem nutrido (ASG A) & 54 & 56,2 \\
\hline $\begin{array}{l}\text { Desnutrição moderada ou suspeita } \\
\text { (ASG B) }\end{array}$ & 28 & 29,2 \\
\hline Gravemente desnutrido (ASG C) & 14 & 14 , \\
\hline
\end{tabular}

${ }^{a}$ Não necessita de intervenção nutricional no momento. ${ }^{\text {b }}$ Educação nutricional para o paciente e família. ${ }^{c}$ Requer intervenção nutricional realizada pelo nutricionista, em conjunto com enfermeiro ou médico como indicado pelos sintomas. ${ }^{\mathrm{d}}$ Necessidade de intervenção nutricional crítica. vada em $17,7 \%$. Dos indivíduos que apresentaram algum grau de depressão (leve ou severa), a maioria eram mulheres (Tabela 2).

A comparação das pontuações obtidas no MEEM, GDS-15 e ASG-PPP segundo o sexo identificou diferença significativa na GDS-15, com maior pontuação na escala para o sexo feminino, indicando um maior número de sintomas depressivos (Tabela 3).

Inicialmente, verificou-se por meio da análise bivariada, a associação entre desnutrição e o menor tempo de diagnóstico, a doença em estágio avançado, não ter realizado cirurgia oncológica, estar em tratamento por quimioterapia, apresentar dois ou mais sintomas de impacto nutricional pela avaliação da ASG-PPP, o déficit cognitivo e funcional (Tabela 4).

O modelo final de associação entre a desnutrição e as variáveis independentes, elegíveis no modelo bivariado, apontou como fatores de risco independentemente associados à desnutrição em idosos em tratamento oncológico o déficit funcional, a presença de dois ou mais sintomas de impacto nutricional e o tratamento por quimioterapia (Tabela 5).

\section{Discussão}

A avaliação nutricional dos idosos em tratamento oncológico evidenciou uma elevada frequência de desnutrição grave ou moderada e de necessidade de intervenção nutricional crítica. Os resultados confirmam um panorama de risco também observado por outros estudos realizados com indivíduos com câncer que utilizaram o mesmo instrumento para o diagnóstico nutricional, nos quais a prevalência de desnutrição grave/ moderada variou de $43,4 \%{ }^{27}$ a $58,4 \%{ }^{28}$ e a necessidade de intervenção crítica alcançou 59,5\% ${ }^{29}$.

A presença de dificuldades para se alimentar foi relatada por $65,6 \%$ dos idosos e os sintomas mais mencionados foram boca seca, constipação intestinal, falta de apetite e saciedade precoce. Segundo dados do Instituto Nacional de Câncer ${ }^{30}$, a perda de apetite está presente de $65 \%$ a $85 \%$ dos pacientes com doença avançada, enquanto náuseas e vômitos podem alcançar até $60 \%$ dos indivíduos em tratamento. As causas são multifatoriais, relacionando-se, principalmente, a questões associadas à localização, evolução tumoral e a terapia medicamentosa ${ }^{31}$. Ressalta-se que em $59,1 \%$ dos idosos foi observada a presença de pelo menos dois sintomas de impacto nutricional, que, na análise multivariada, mostrou-se 


\begin{tabular}{|c|c|c|c|c|c|}
\hline Parâmetro & $\begin{array}{r}\text { Homens } \\
\mathbf{n}(\%)\end{array}$ & $\begin{array}{c}\text { Mulheres } \\
\mathbf{n}(\%)\end{array}$ & Total & $\%^{*}$ & $\mathbf{p}^{* *}$ \\
\hline Função cognitiva - MEEM & & & & & 0,68 \\
\hline Sem déficit cognitivo & $30(51,7)$ & $28(48,3)$ & 58 & 60,4 & \\
\hline Com déficit cognitivo & $18(47,4)$ & $20(52,6)$ & 38 & 39,6 & \\
\hline Depressão - GDS-15 & & & & & 0,06 \\
\hline Sem depressão & $43(54,4)$ & $36(45,6)$ & 79 & 82,3 & \\
\hline Com depressão & $5(29,4)$ & $12(70,6)$ & 17 & 17,7 & \\
\hline Leve & $5(35,7)$ & $9(64,3)$ & 14 & 14,6 & \\
\hline Severa & $0(0,0)$ & $3(100,0)$ & 3 & 3,1 & \\
\hline
\end{tabular}

MEEM: Miniexame do Estado Mental; GDS-15: Escala de Depressão Geriátrica versão com 15 itens.

"Em relação à amostra total. ${ }^{* *}$ Teste do qui-quadrado.

Tabela 3. Pontuação no Miniexame do Estado Mental, na Escala de Depressão Geriátrica e na Avaliação Subjetiva Global Produzida pelo Paciente de idosos em tratamento oncológico, segundo o sexo. Ponte Nova, 2012.

\begin{tabular}{|c|c|c|c|c|c|}
\hline \multirow[b]{2}{*}{ Parâmetro } & \multicolumn{2}{|c|}{ Total $(n=96)$} & \multirow{2}{*}{$\begin{array}{c}\text { Masculino }(\mathrm{n}=48) \\
\text { Média }(\mathrm{DP}) \\
\text { MD (mín-máx) }\end{array}$} & \multirow{2}{*}{$\begin{array}{c}\text { Feminino }(\mathrm{n}=48) \\
\text { Média (DP) } \\
\text { MD (mín-máx) }\end{array}$} & \multirow[b]{2}{*}{$\mathbf{p}^{*}$} \\
\hline & $\begin{array}{l}\text { Média } \\
\text { (DP) }\end{array}$ & $\begin{array}{c}\text { MD } \\
\text { (mín-máx) }\end{array}$ & & & \\
\hline MEEM & $\begin{array}{l}22,2 \\
(4,2)\end{array}$ & $\begin{array}{c}22,0 \\
(11-30)\end{array}$ & $\begin{array}{c}22,3(4,4) \\
23,0(11-30)\end{array}$ & $\begin{array}{c}22,1(3,9) \\
21,0(15-30)\end{array}$ & 0,564 \\
\hline GDS-15 & $\begin{array}{c}3,7 \\
(2,5)\end{array}$ & $\begin{array}{c}3,0 \\
(0-13)\end{array}$ & $\begin{array}{l}2,9(1,7) \\
3,0(0-7)\end{array}$ & $\begin{array}{c}4,4(2,9) \\
4,0(0-13)\end{array}$ & 0,017 \\
\hline ASG-PPP & $\begin{array}{l}10,4 \\
(7,0)\end{array}$ & $\begin{array}{c}8,0 \\
(1-27)\end{array}$ & $\begin{array}{c}9,9(7,2) \\
8,0(2-27)\end{array}$ & $\begin{array}{c}10,9(6,9) \\
10,0(1-26)\end{array}$ & 0,407 \\
\hline
\end{tabular}

DP: desvio-padrão; MD: mediana; mín: mínimo; máx: máximo. MEEM: pontuação no Miniexame do Estado Mental; GDS-15: pontuação na Escala de Depressão Geriátrica, versão com 15 itens; ASG-PPP: pontuação de triagem na Avaliação Subjetiva Global Produzida pelo Paciente. Teste de Mann-Whitney.

Tabela 4. Prevalência e Razão de Prevalência de desnutrição segundo condições sociodemográficas, clínicas e de saúde em idosos em tratamento oncológico. Ponte Nova, 2012.

\begin{tabular}{|c|c|c|c|c|}
\hline Variáveis & $\begin{array}{c}\text { Total } \\
\mathbf{n}\end{array}$ & $\begin{array}{c}\text { Desnutrição } \\
\text { n (\%) }\end{array}$ & $\begin{array}{l}\text { RP bruta } \\
\text { (IC 95\%) }\end{array}$ & $\mathbf{p}$ \\
\hline \multicolumn{5}{|l|}{ Sociodemográficas } \\
\hline Sexo & & & & 0,410 \\
\hline Masculino & 48 & $19(39,6)$ & 1 & \\
\hline Feminino & 48 & $23(47,9)$ & $1,18(0,79-1,76)$ & \\
\hline \multicolumn{5}{|l|}{ Faixa etária } \\
\hline $60-69$ anos & 43 & $19(44,2)$ & 1 & \\
\hline $70-79$ anos & 38 & $16(42,1)$ & $0,95(0,59-1,53)$ & 0,852 \\
\hline 80 ou mais & 15 & $7(46,7)$ & $1,06(0,56-2,01)$ & 0,868 \\
\hline Anos de estudo* & & & & 0,243 \\
\hline$>3$ & 43 & $16(37,2)$ & 1 & \\
\hline$\leq 3$ & 53 & $26(49,1)$ & $1,24(0,86-1,77)$ & \\
\hline Renda per capita* $(\mathrm{n}=89)$ & & & & 0,357 \\
\hline$>466,00$ & 46 & $18(39,1)$ & 1 & \\
\hline$\leq 466,00$ & 43 & $21(48,8)$ & $1,22(0,80-1,88)$ & \\
\hline Presença de companheiro(a) & & & & 0,135 \\
\hline $\operatorname{Sim}^{\mathrm{a}}$ & 54 & $20(37,0)$ & 1 & \\
\hline Não ${ }^{b}$ & 42 & $22(52,4)$ & $1,41(0,90-2,22)$ & \\
\hline Presença de cuidador & & & & 0,233 \\
\hline Sim & 82 & $38(46,3)$ & 1 & \\
\hline Não & 14 & $4(28,6)$ & $0,51(0,17-1,53)$ & \\
\hline
\end{tabular}


Tabela 4. continuação

\begin{tabular}{|c|c|c|c|c|}
\hline Variáveis & $\begin{array}{c}\text { Total } \\
\mathbf{n}\end{array}$ & $\begin{array}{c}\text { Desnutrição } \\
\mathbf{n}(\%)\end{array}$ & $\begin{array}{l}\text { RP bruta } \\
\text { (IC 95\%) }\end{array}$ & $\mathbf{p}$ \\
\hline \multicolumn{5}{|l|}{ Clínicas } \\
\hline Tempo de diagnóstico ${ }^{*}$ & & & & 0,041 \\
\hline$\geq 12,87$ meses & 48 & $16(33,3)$ & 1 & \\
\hline$<12,87$ meses & 48 & $26(54,2)$ & $1,52(1,02-2,27)$ & \\
\hline Presença de metástase & & & & 0,719 \\
\hline Não & 50 & $21(42,0)$ & 1 & \\
\hline Sim & 46 & $21(45,7)$ & $1,08(0,71-1,64)$ & \\
\hline Estadiamento & & & & 0,023 \\
\hline $\mathrm{I} / \mathrm{II}$ & 16 & $3(18,8)$ & 1 & \\
\hline III/IV & 80 & $39(48,8)$ & $1,2(1,03-1,45)$ & \\
\hline Cirurgia oncológica prévia & & & & 0,003 \\
\hline Sim & 49 & $14(28,6)$ & 1 & \\
\hline Não & 47 & $28(59,6)$ & $1,89(1,24-2,89)$ & \\
\hline Tratamento atual & & & & 0,000 \\
\hline Hormonioterapia & 32 & $4(12,5)$ & 1 & \\
\hline Quimioterapia & 64 & $38(59,4)$ & $1,88(1,34-2,52)$ & \\
\hline Radioterapia prévia & & & & 0,213 \\
\hline Sim & 34 & $12(35,3)$ & 1 & \\
\hline Não & 62 & $30(48,4)$ & $1,21(0,90-1,62)$ & \\
\hline Tempo de tratamento* & & & & 0,101 \\
\hline$\geq 5$ meses & 48 & $17(35,4)$ & 1 & \\
\hline$<5$ meses & 48 & $25(52,1)$ & $1,40(0,94-2,08)$ & \\
\hline No de sintomas de impacto nutricional & & & & 0,002 \\
\hline$<2$ & 30 & $3(10,0)$ & 1 & \\
\hline$\geq 2$ & 66 & $39(59,1)$ & $5,90(1,97-17,71)$ & \\
\hline \multicolumn{5}{|l|}{ Condições de saúde } \\
\hline Depressão & & & & 0,404 \\
\hline Ausência & 79 & $33(41,8)$ & 1 & \\
\hline Presença ${ }^{\mathrm{c}}$ & 17 & $9(52,9)$ & $1,45(0,61-3,44)$ & \\
\hline Função cognitiva & & & & 0,027 \\
\hline Sem déficit & 58 & $20(34,5)$ & 1 & \\
\hline Com déficit & 38 & $22(57,9)$ & $1,77(1,07-2,93)$ & \\
\hline Capacidade funcional $^{\mathrm{d}}$ & & & & 0,000 \\
\hline Sem déficit & 39 & $4(10,3)$ & 1 & \\
\hline Com déficit & 57 & $38(66,7)$ & $6,5(2,51-16,82)$ & \\
\hline Autopercepção da saúde & & & & 0,316 \\
\hline Positiva $^{\mathrm{e}}$ & 49 & $19(38,8)$ & 1 & \\
\hline Negativa $^{\mathrm{f}}$ & 47 & $23(48,9)$ & $1,23(0,82-1,85)$ & \\
\hline No de medicamentos ${ }^{*}$ & & & & 0,793 \\
\hline$\leq 2$ & 58 & $26(44,8)$ & 1 & \\
\hline$>2$ & 38 & $16(42,1)$ & $0,93(0,56-1,55)$ & \\
\hline Presença de comorbidades & & & & 0,889 \\
\hline Não & 29 & $13(44,8)$ & 1 & \\
\hline Sim & 67 & $29(43,3)$ & $0,97(0,39-1,10)$ & \\
\hline
\end{tabular}

RP: Razão de Prevalência; IC: Intervalo de Confiança. * categorização segundo a mediana da amostra, uma vez que as variáveis

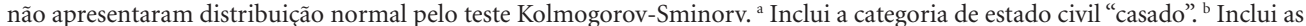
categorias de estado civil "solteiro", "viúvo" e "divorciado". ' Inclui as categorias "leve" e "severa". 'Segundo critérios da Avaliação Subjetiva Global Produzida pelo Paciente, foi considerada ausência de déficit funcional os idosos que relataram capacidade funcional no último mês como "normal, sem limitação". " Inclui as categorias "excelente", "muito boa" e "boa". ${ }^{\mathrm{f}}$ Inclui as categorias "regular" e "ruim". g Com exceção dos medicamentos utilizados para o câncer. 
Tabela 5. Modelo final da regressão múltipla dos fatores associados à desnutrição em idosos em tratamento oncológico. Ponte Nova, 2012.

\begin{tabular}{lcc}
\hline \multicolumn{1}{c}{ Variáveis } & RP ajustada (IC 95\%) & $\mathbf{p}$ \\
\hline $\begin{array}{l}\text { Capacidade funcional } \\
\text { Sem déficit }\end{array}$ & $\mathbf{0 , 0 1 9}$ \\
$\quad$ Com déficit & $3,40(1,23-9,45)$ & \\
$\begin{array}{l}\text { No de sintomas de } \\
\text { impacto nutricional }\end{array}$ & $\mathbf{0 , 0 4 5}$ \\
$\quad \begin{array}{l}\text { Menos que 2 } \\
\text { 2 ou mais }\end{array}$ & 1 & \\
$\begin{array}{l}\text { Tipo de tratamento } \\
\text { Hormonioterapia } \\
\text { Quimioterapia }\end{array}$ & $2,22(1,03-10,10)$ & $\mathbf{0 , 0 2 3}(1,16-7,56)$ \\
\end{tabular}

RP: Razão de Prevalência. IC: Intervalo de Confiança.

como um fator de risco independentemente associado à desnutrição.

A prevalência de depressão identificada pela Escala de Depressão Geriátrica (GDS) foi de $17,7 \%$, sendo a pontuação significativamente superior entre as mulheres. Segundo Croyle e Rowland $^{32}$, cerca de $10 \%$ a $25 \%$ dos indivíduos com câncer apresentarão, além da reação esperada frente à doença, episódio de depressão maior e/ou de ansiedade.

Em estudos que avaliaram a prevalência de depressão em indivíduos com câncer em diferentes contextos clínicos, foi observada prevalência de $14 \%$ em pacientes ambulatoriais ${ }^{33}$, semelhante ao observado no presente estudo também com pacientes ambulatoriais, $28 \%$ em unidade de cuidados paliativos ${ }^{34}$ e de 20 a $25 \%$ em indivíduos hospitalizados $^{35}$. Em revisão de literatura com estudos que avaliaram a depressão em pacientes com câncer avançado e dor, Juver e Verçosa ${ }^{36}$ observaram prevalências em torno de $50 \%$ a $60 \%$.

O maior número de sintomas depressivos observado no sexo feminino corrobora um dado consistente em estudos que avaliaram a epidemiologia da depressão ${ }^{37,38}$, que também foi verificado em indivíduos com câncer ${ }^{39}$. Em relação aos possíveis fatores que poderiam justificar essa diferença, destacam-se: maior sensibilidade a experiências traumáticas na infância e adolescência, violência doméstica, ansiedade, sobrecarga de funções e papéis sociais e maior vulnerabilidade a eventos estressantes ${ }^{40}$. Linhares et al. ${ }^{41}$ destacam a influência da viuvez e de perdas familiares.

A depressão repercute de forma bastante negativa na qualidade de vida, leva à não adesão ao tratamento proposto e prolonga as internações hospitalares $^{42}$. É usualmente pouco investigada e subdiagnosticada na população idosa, sendo o reconhecimento ativo e o tratamento de quadros depressivos partes relevantes no cuidado ao indivíduo com câncer, podendo contribuir favoravelmente para melhorar atitudes diante da enfermidade e melhorar o bem-estar ${ }^{43}$.

Dos idosos avaliados, 39,6\% apresentaram déficit cognitivo, sem diferença entre os sexos na pontuação do Mini Exame do Estado Mental (MEEM). Foi demonstrado que de $25 \%$ a $50 \%$ dos idosos com câncer podem apresentar pontuação indicativa de anormalidades cognitivas no $\mathrm{MEEM}^{44}$, estimativa confirmada neste estudo. A função cognitiva tem implicações práticas importantes e consequências diretas sobre a qualidade de vida, especialmente em idosos com câncer. Na presença de declínio de memória, o paciente pode ter dificuldade de compreender e recordar as instruções do tratamento, de lembrar os sinais e sintomas da doença e os efeitos colaterais da terapia que merecem atenção médica ${ }^{21 .}$

Os fatores independentemente associados à desnutrição nos idosos foram o comprometimento funcional, a presença de dois ou mais sintomas de impacto nutricional e o tratamento por quimioterapia.

O declínio funcional ocorre na população idosa, com sintomas inespecíficos, apresentação atípica, curso insidioso e engloba manifestações físicas, psicológicas, sociais e funcionais ${ }^{45}$. Ocasiona maior vulnerabilidade e dependência, contribuindo para a diminuição do bem-estar e da qualidade de vida dos idosos ${ }^{46}$. A capacidade funcional é dimensionada em termos da habilidade e independência para realizar determinadas atividades ${ }^{47}$. Em estudos com idosos, estes geralmente são avaliados pelas atividades de vida diária (AVD), pelas atividades instrumentais de vida diária (AIVD) e pela mobilidade física ${ }^{48}$. Como a ASG-PPP inclui a avaliação da capacidade funcional, representa uma nova possibilidade para esta investigação. Neste sentido, mais estudos são necessários para verificar a concordância da avaliação da funcionalidade pela ASG-PPP com os instrumentos tradicionais.

Foi demonstrada a associação entre o comprometimento funcional e a desnutrição na admissão hospitalar ${ }^{49}$, relação também verificada em idosos institucionalizados ${ }^{50}$. No estudo de Saka et al. ${ }^{51}$, com 410 idosos, $84 \%$ dos pacientes idosos funcionalmente dependentes apresentaram pior estado nutricional. Em um estudo populacional nos Estados Unidos, com 9745 idosos, dos quais 1647 relataram a presença de câncer, 
indivíduos com a doença relataram maior dificuldade para caminhar, tomar banho, sentar e levantar de uma cadeira ou cama, realizar tarefas domésticas, fazer compras e preparar refeições quando comparados aos idosos sem a doença ${ }^{52}$. Ressalta-se que a maioria dos estudos disponíveis na literatura até o momento utilizou outras formas de diagnóstico nutricional, tais como antropometria. A metodologia distinta utilizada no presente estudo para a mensuração e a classificação da variável dependente (desnutrição) poderia alterar a identificação de associações. No entanto, conforme demonstrado, o déficit cognitivo também se associou à desnutrição quando esta foi avaliada pela ASG-PPP, atual recomendação do Instituto Nacional do Câncer.

Em relação à presença de sintomas de impacto nutricional, já é bem estabelecido sua interferência na ingestão de alimentos, no aproveitamento dos nutrientes e, consequentemente, no desenvolvimento de estados carenciais ${ }^{53}$. Destaca-se, entretanto, que a presença de apenas dois sintomas ou mais aumentou em mais de três vezes a prevalência de desnutrição, independentemente de outras condições. A identificação deste impacto alerta para a relevância do manejo nutricional intensivo, do rastreamento constante e da intervenção precoce.

O tratamento por quimioterapia, em comparação à hormonioterapia, também foi identificado como determinante da desnutrição. A hormonioterapia consiste no uso de substâncias semelhantes ou inibidoras de hormônios para tratar as neoplasias que são dependentes desses e, embora seja considerado um tipo de tratamento quimioterápico, apresenta mecanismos de ação e efeitos colaterais diferentes em relação aos quimioterápicos antineoplásicos ${ }^{54}$. Por atuar somente sobre as células que expressam receptores hormonais, principalmente no tratamento dos tumores de mama, próstata e endométrio, seus efeitos colaterais são menos intensos e com menor toxicidade ${ }^{55}$.

Em revisão sobre os efeitos dos medicamentos utilizados na hormonioterapia em mulheres, houve destaque para fogachos, artralgia, retenção hídrica, disfunção sexual, cefaleia, edema e ganho de peso. Embora alguns medicamentos possam estar associados a náuseas/vômitos, foi possível observar que a maioria dos sintomas citados não exerceu impacto direto no consumo de alimentos quando comparados à quimioterapia ${ }^{56}$. Apesar das diferenças terapêuticas serem bem estabelecidas, sua interferência distinta na ocorrência de desnutrição ainda não havia sido demonstrada quantitativamente na literatura, o que evidencia a importância dos presentes resultados.

A relação de associação obtida em estudos transversais não possibilita estabelecer uma relação temporal entre os eventos e uma relação causa-efeito ${ }^{57}$, mas indicam parâmetros relevantes que ao se associarem à maior prevalência do desfecho (desnutrição), alertam profissionais de saúde para grupos potencialmente de maior risco. Neste sentido, destaca-se que dos três fatores que se associaram à maior prevalência de desnutrição nos idosos, dois são parâmetros investigados pela ASG-PPP, reforçando sua importância e abrangência na avaliação nutricional de idosos em tratamento oncológico. A ciência que envolve a atenção a idosos com câncer cresce na medida em que se comprova a aplicabilidade de um novo método de avaliação nutricional, na quantificação e alerta da influencia de apenas poucos sintomas, do tipo de tratamento e do déficit funcional na prevalência de desnutrição.

\section{Considerações finais}

O déficit cognitivo e a presença de depressão são importantes aspectos a serem avaliados no idoso com câncer. A desnutrição moderada ou grave foi identificada em cerca de dois a cada cinco avaliados, sendo que a presença de déficit funcional, de dois ou mais sintomas de impacto nutricional e o tratamento por quimioterapia apresentaram-se como fatores de risco independentemente associados à desnutrição.

Reforça-se a importância da participação do nutricionista na assistência ao indivíduo com câncer, por meio do diagnóstico e da intervenção nutricional com ênfase no manejo dos sintomas. Destaca-se, também, a importância de uma abordagem ampla e integral, que considere aspectos como a presença de depressão, o déficit cognitivo e funcional, uma vez que repercutem diretamente na saúde e na qualidade de vida.

\section{Colaboradores}

CA Santos participou da concepção, delineamento, coleta e análise dos dados e na redação do artigo. AQ Ribeiro e COB Rosa trabalharam na interpretação dos dados, na revisão crítica e na aprovação da versão a ser publicada. RCL Ribeiro participou da concepção, delineamento, interpretação dos dados, na revisão crítica e na aprovação da versão a ser publicada. 


\section{Referências}

1. Silva MM, Silva VH. Envelhecimento: importante fator de risco para o câncer. Arq Med ABC 2005; 30(1):11-18.

2. Instituto Nacional de Câncer José Alencar Gomes da Silva (Inca). Coordenação Geral de Ações Estratégicas. Coordenação de Prevenção e Vigilância. Estimativa 2012: incidência de câncer no Brasil. Rio de Janeiro: Inca; 2011.

3. Lichtman SM. Guidelines for the treatment of elderly cancer patients. Cancer Control 2003; 10(6):445-453.

4. Ravasco P, Monteiro-Grillo I, Vidal PM, Camilo ME. Nutritional deterioration in cancer: the role of disease and diet. Clin Oncol (R Coll Radiol) 2003; 15(8):443450 .

5. Marín Caro MM, Laviano A, Pichard C. Nutritional intervention and quality of life in adult oncology patients. Clin Nutr 2007; 26(3):289-301.

6. Gonzalez MC, Borges LR, Silveira DH, Assunção MCF, Orlandi SP. Validação da versão em português da Avaliação Subjetiva Global Produzida pelo Paciente. Rev Bras Nutr Clin 2010; 25(2):102-108.

7. Bozzetti F. Nutritional aspects of the cancer/aging interface. J Geriatr Oncol 2011; 2(3):177-186.

8. Cavalini LT, Chor D. Inquérito sobre hipertensão arterial e décifit cognitivo em idosos de um serviço de geriatria. Rev Bras Epidemiol 2003; 6(1):7-17.

9. Ferrari JL, Dalacorte RR. Uso da Escala de Depressão Geriátrica de Yesavage para avaliar a prevalência de depressão em idosos hospitalizados. Scientia Medica 2007; 17(1):3-8.

10. Brant JM. Palliative care for adults across the cancer trajectory: from diagnosis to end of life. Semin Oncol Nurs 2010; 26(4):222-230.

11. Khasraw M, Posner JB. Neurological complications of systemic cancer. Lancet Neurol 2010; 9(12):1214-1227.

12. Stilley CS, Bender CM, Dunbar-Jacob J, Sereika S, Ryan $\mathrm{CM}$. The impact of cognitive function on medication management: three studies. Health Psychol 2010; 29(1):50-55.

13. Watson R, Green SM. Feeding and dementia: a systematic literature review. J Adv Nurs 2006; 54(1):86-93.

14. Castelo MS, Coelho Filho JM, Siqueira Neto JI, Noleto JCS, Lima JWO. Escala de Depressão Geriátrica com quatro itens: um instrumento válido para rastrear depressão em idosos em nível primário de saúde. Rev Bras Geriatr Gerontol 2007; 1(1):28-33.

15. Skarstein J, Aass N, Fosså SD, Skovlund E, Dahl AA. Anxiety and depression in cancer patients: relation between the Hospital Anxiety and Depression Scale and the European Organization for Research and Treatment of Cancer Core Quality of Life Questionnaire. J Psychosom Res 2000; 49(1):27-34.

16. Langa KM, Valenstein MA, Fendrick AM, Kabeto MU, Vijan S. Extent and cost of informal caregiving for older americans with symptoms of depression. Am J Psychiatry 2004; 161(5):857-863.

17. Balducci L. Geriatric oncology: challenges for the new century. Eur J Cancer 2000; 36(14):1741-1754.

18. Katona C, Livingston G, Manela M, Leek C, Mullan E, Orrell M, D'Ath P, Zeitlin D. The symptomatology of depression in the elderly. Int Clin Psychopharmacol 1997; 12(Supl. 7):S19-23.

19. Barrero SAP. Factores de riesgo suicida en el anciano. Cien Saude Colet 2012; 17(8):2011-2016.
20. German L, Feldblum I, Bilenko N, Castel H, Harman -Boehm I, Shahar DR. Depressive symptoms and risk for malnutrition among hospitalized elderly people. $J$ Nutr Health Aging 2008; 12(5):313-318.

21. Yoshimura K, Yamada M, Kajiwara Y, Nishiguchi S, Aoyama T. Relationship between depression and risk of malnutrition among community-dwelling young-old and old-old elderly people. Aging Ment Health 2013; 17(4):456-460.

22. Brunello A, Sandri R, Extermann M. Multidimensional geriatric evaluation for older cancer patients as a clinical and research tool. Cancer Treat Rev 2009; 35(6):487492.

23. Folstein MF, Folstein SE, McHugh PR. Mini-Mental State: a practical method for grading the cognitive state of patients for the chinican. J Psychiatr Res 1975; 12(3):189-198.

24. Brucki SMD, Nitrini R, Caramelli P, Bertolucci PHF, Okamoto IH. Sugestões para o uso do Mini-Exame do Estado Mental no Brasil. Arq Neuropsiquiatr 2003; 61(3-B):777-781.

25. Almeida OP, Almeida SA. Confiabilidade da versão brasileira da Escala de Depressão Geriátrica (GDS) versão reduzida. Arq Neuropsiquiatr 1999; 57(2-B):421-426.

26. Yesavage JA, Brink TL, Rose TL, Lum O, Huang V, Adey $\mathrm{M}$, Leirer VO. Development and validation of a geriatric depression screening scale: a preliminary report. $J$ Psychiatr Res 1983; 17(1):37-49.

27. Nourissat A, Vasson MP, Merrouche Y, Bouteloup C, Goutte M, Mille D, Jacquin JP, Collard O, Michaud P, Chauvin F. Relationship between nutritional status and quality of life in patients with cancer. Eur J Cancer 2008; 44(9):1238-1242.

28. Brito LF, Silva LS, Fernandes DD, Pires RA, Nogueira ADR, Souza CL, Cardoso LGV. Perfil nutricional de pacientes com câncer assistidos pela Casa de Acolhimento ao Paciente Oncológico do Sudoeste da Bahia. Rev Bras Cancerol 2012; 58(2):163-171.

29. Segura A, Pardo J, Jara C, Zugazabeitia L, Carulla J, de las Peñas R, García-Cabrera E, Azuara ML, Casadó J, Goméz-Candela C. An epidemiological evaluation of the prevalence of malnutrition in Spanish patients with locally advanced or metastatic cancer. Clinical Nutrition 2005; 24(5):801-814.

30. Instituto Nacional de Câncer (Inca). Cuidados paliativos oncológicos: controle de sintomas. Rio de Janeiro: Inca; 2001.

31. Dias VM, Coelho SC, Ferreira FMB, Vieira GBS, Cláudio MM, Silva PDG. O grau de interferência dos sintomas gastrintestinais no estado nutricional do paciente com câncer em tratamento quimioterápico. Rev Bras Nutr Clin 2005; 21(2):104-110.

32. Croyle RT, Rowland JH. Mood disorders and cancer: a National Cancer Institute perspective. Biol Psychiatry 2003; 54(3):191-194.

33. Berard RM, Boermeester F, Viljoen G. Depressive disorders in an outpatient oncology setting: prevalence, assessment, and management. Psychooncology 1998; 7(2):112-120

34. Ciaramella A, Poli P. Assessment of depression among cancer patients: the role of pain, cancer type and treatment. Psychooncology 2001; 10(2):156-165. 
35. Krishnan KR, Delong M, Kraemer H, Carney R, Spiege D, Gordon C, McDonald W, Dew M, Alexopoulos G, Buckwalter K, Cohen PD, Evans D, Kaufmann PG, Olin J, Otey E, Wainscott C. Comorbidity of depression with other medical diseases in the elderly. Biol Psychiatry 2002; 52(6):559-588

36. Juver JPS, Verçosa N. Depressão em pacientes com dor no câncer avançado. Rev Bras Anestesiol 2008; 58(3): 287-298.

37. Cole MG, Dendukuri N. Risk factors for depression among elderly community subjects: a systematic review and meta-analysis. Am J Psychiatry 2003; 160(6):11471156.

38. Gazalle FK, Lima MS, Tavares BF, Hallal PC. Sintomas depressivos e fatores associados em população idosa no Sul do Brasil. Rev Saude Publica 2004; 38(3):365-371.

39. Massie MJ. Prevalence of depression in patients with cancer. J Natl Cancer Inst Monogr 2004; (32):57-71.

40. Grover S, Dutt A, Avasthi A. An overview of Indian research in depression. Indian J Psychiatry 2010; 52(1): 178-188.

41. Linhares CRC, Coelho VLD, Guimarães RM, Campos APM, Carvalho NT. Perfil da clientela de um ambulatório de geriatria do Distrito Federal. Psicol Reflex Crit 2003; 16(2):319-326

42. Teng CT, Humes EC, Demetrio FN. Depressão e comorbidades clínicas. Rev Psiq Clín 2005; 32(3):149-159.

43. Raison CL, Miller AH. Depression in cancer: new developments regarding diagnosis and treatment. Biol Psychiatry 2003; 54(3):283-294.

44. Extermann M, Hurria A. Comprehensive geriatric assessment for older patients with cancer. J Clin Onco 2007; 25(14):1824-1831.

45. Trelha CS, Nakaoski T, Franco SS, Dellaroza MSG, Yamada KN, Cabrera M, Mesas AE, Gaetan CCB. Capacidade funcional de idosos restritos ao domicílio, do conjunto Ruy Virmond Carnascialli, Londrina/PR. Semina Cienc Biol Saude 2005; 26(1):37-46.

46. Alves LC, Leite IC, Machado CJ. Conceituando e mensurando a incapacidade funcional da população idosa: uma revisão de literatura. Cien Saude Colet 2008 13(4):1199-1207.

47. Lima-Costa MF, Barreto SM, Giatti L. Condições de saúde, capacidade funcional, uso de serviços de saúde e gastos com medicamentos da população idosa brasileira: um estudo descritivo baseado na Pesquisa Nacional por Amostra de Domicílios. Cad Saude Publica 2003; 19(3):735-743.
48. Alves LC, Leite IC, Machado CJ. Conceituando e mensurando a incapacidade funcional da população idosa: uma revisão de literatura. Cien Saude Colet 2008; 13(4):1199-1207.

49. Amaral TF, Matos LC, Teixeira MA, Tavares MM, Alvares L, Antunes A. Undernutrition and associated factors among hospitalized patients. Clinical Nutrition 2010; 29(5):580-585.

50. Suominen M, Muurinen S, Routasalo P, Soini H, Suur -Uski I, Peiponen A, Finne-Soveri H, Pitkala KH. Malnutrition and associated factors among aged residents in all nursing homes in Helsinki. Eur J Clin Nutr 2005; 59(4):578-583.

51. Saka B, Kaya O, Ozturk GB, Erten N, Karan MA. Malnutrition in the elderly and its relationship with other geriatric syndromes. Clinical Nutrition 2010; 29(6):745-748.

52. Stafford RS, Cyr PL. The impact of cancer on the physical function of the elderly and their utilization of health care. Cancer 1997; 80(10):1973-1980.

53. Candela CG, Peña GM, Cos Blanco AI, Rosado CI, Rabaneda RC. Evaluación del estado nutricional en el paciente oncológico. Suporte Nutricional en el Paciente Oncológico 2004; 4(1):43-56.

54. Instituto Nacional de Câncer José Alencar Gomes da Silva. Coordenação Geral de Ações Estratégicas. Coordenação de Educação. $A B C$ do câncer: abordagens básicas para o controle do câncer. $2^{\text {a }}$ ed. Rio de Janeiro: INCA; 2012.

55. Bonassa EMA, Gato MIR. Terapia hormonal. In: Bonassa EMA, Gato MIR. Terapêutica oncológica para enfermeiros e farmacêuticos. $4^{\text {a }}$ ed. São Paulo: Atheneu; 2012. p. 25-27.

56. Leal JHS, Cubero D, Del Giglio A. Hormonioterapia paliativa em câncer de mama: aspectos práticos e revisão da literatura. Rev Bras Clin Med 2010; 8(4):338-343.

57. Bastos JLD, Duquia RP. Um dos delineamentos mais empregados em epidemiologia: estudo transversal. Scientia Medica 2007; 17(4):229-232.

Artigo apresentado em 26/05/2014 Aprovado em 26/08/2014

Versão final apresentada em 28/08/2014 